\title{
CORRECTION
}

\section{Author Correction: PTEN-L is a novel protein phosphatase for ubiquitin dephosphorylation to inhibit PINK1-Parkin-mediated mitophagy}

Liming Wang ${ }^{1}$, Yik-Lam Cho ${ }^{1}$, Yancheng Tang ${ }^{2}$, Jigang Wang ${ }^{1}$, Jung-Eun Park ${ }^{3}$, Yajun Wu ${ }^{4}$, Chunxin Wang (D) $^{5}$, Yan Tong ${ }^{6}$, Ritu Chawla ${ }^{1}$, Jianbin Zhang ${ }^{1,7}$, Yin Shi ${ }^{1}$, Shuo Deng ${ }^{1}$, Guang Lu ${ }^{1}$, Yihua Wu ${ }^{8}$, Hayden Weng-Siong Tan ${ }^{1,9}$, Pornteera Pawijit ${ }^{1}$, Grace Gui-Yin Lim ${ }^{10}$, Hui-Ying Chan ${ }^{1,9}$, Jingzi Zhang ${ }^{11}$, Lei Fang ${ }^{11}$, Hanry Yu ${ }^{1,12,13}$, Yih-Cherng Liou ${ }^{6}$, Mallilankaraman Karthik ${ }^{1}$, Boon-Huat Bay ${ }^{4}$, Kah-Leong Lim ${ }^{1,10}$, Siu-Kwan Sze $\mathbb{D}^{3}$, Celestial T. Yap ${ }^{1}$ and Han-Ming Shen ${ }^{1,9}$

Cell Research (2018) 28:872-873; https://doi.org/10.1038/s41422-018-0068-9

Correction to: Cell Research (2018) 0:1-16. https://doi.org/ 10.1038/s41422-018-0056-0; Published online 22 June 2018.

We apologize for three errors that we just found in the paper published online on 22 June 2018. Figure $2 \mathrm{~b}$ was inadvertently duplicated from Supplementary information, Figure S3d. The PTEN-L/PTEN blot in Fig. 4b was inadvertently cropped. The scale bars of ZOOM channel in Supplementary

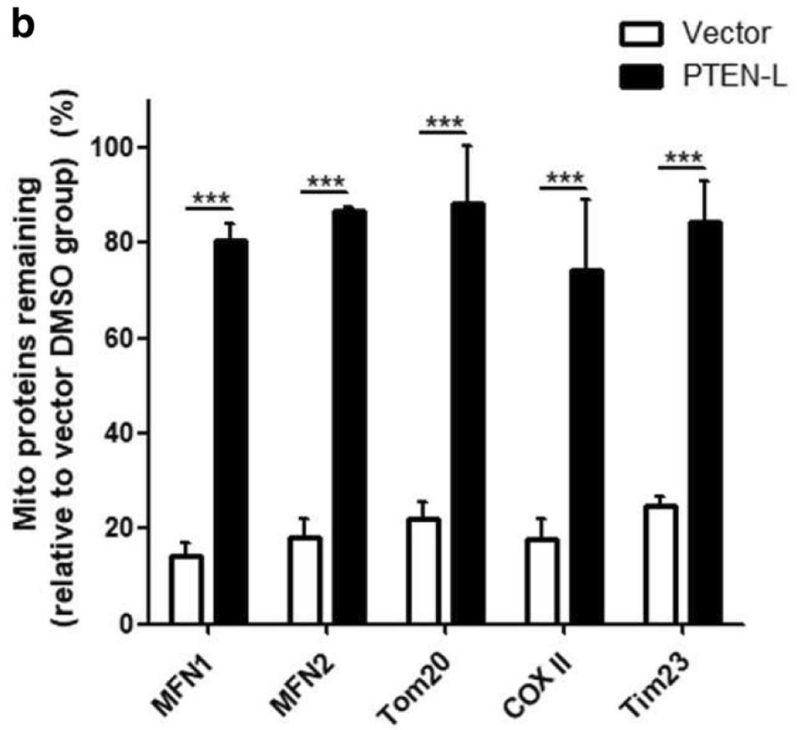

Fig. 2 PTEN-L negatively regulates mitophagy induced by various mitochondria-damaging agents. $\mathbf{b}$ Quantification of mitochondrial proteins from a information, Figure S1f and i, were inadvertently indicated. The corrected version of Figs. $2 \mathrm{~b}$ and $4 \mathrm{~b}$, and the corrected legends of Supplementary information, Figure S1f and $i$, are provided below. No conclusion was affected by these errors, but

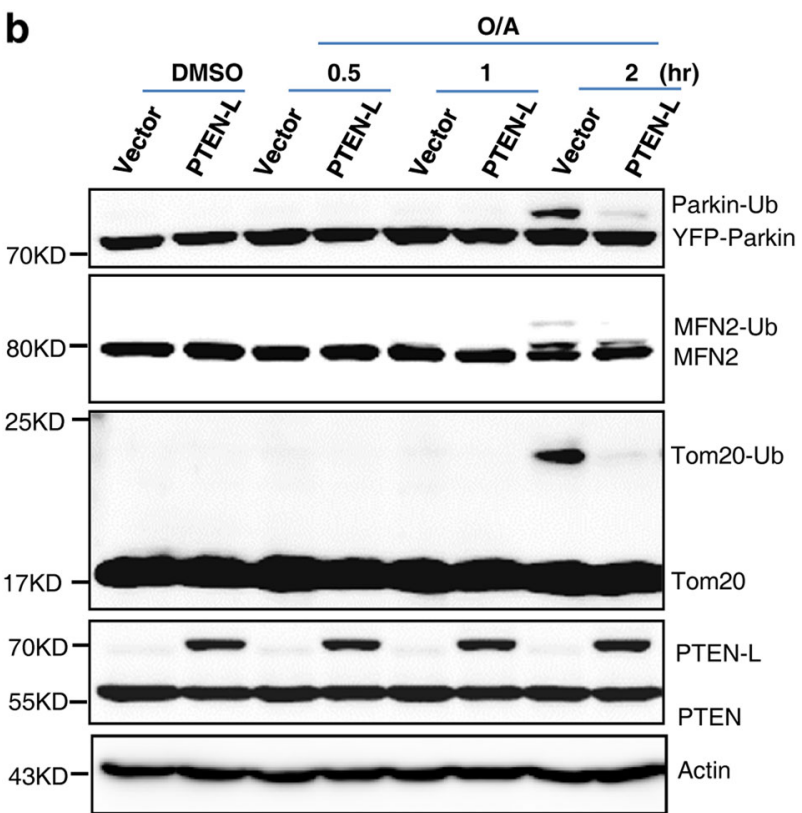

Fig. 4 PTEN-L impairs Parkin E3 ligase activity and reduces pSer65Parkin. b YFP-Parkin-HeLa cells with PTEN-L stable expression or control vector were treated with O/A $(10 \mathrm{nM}$ and $100 \mathrm{nM})$ for indicated hours and the WCLs were analyzed by immunoblotting as indicated

\footnotetext{
${ }^{1}$ Department of Physiology, Yong Loo Lin School of Medicine, National University of Singapore, Singapore, Singapore; ${ }^{2}$ School of Chinese Medicine, Hong Kong Baptist University, Kowloon Tong, Hong Kong, China; ${ }^{3}$ School of Biological Sciences, Nanyang Technological University, Singapore, Singapore; ${ }^{4}$ Department of Anatomy, National University of Singapore, Singapore, Singapore; ${ }^{5}$ Biochemistry Section, Surgical Neurology Branch, National Institute of Neurological Disorders and Stroke, National Institutes of Health, Bethesda, MD 20892, USA; ${ }^{6}$ Department of Biological Sciences, Faculty of Science, National University of Singapore, Singapore, Singapore; ${ }^{7}$ Department of Oncology, Clinical Research Institute, Zhejiang Provincial People's Hospital, People's Hospital of Hangzhou Medical College, Hangzhou, Zhejiang, China; ${ }^{8}$ Department of Toxicology, Zhejiang University School of Public Health, Hangzhou, Zhejiang, China; ${ }^{9}$ Graduate School for Integrative Sciences and Engineering, National University of Singapore, Singapore, Singapore; ${ }^{10}$ National Neuroscience Institute, Singapore, Singapore; ${ }^{11}$ Jiangsu Key Laboratory of Molecular Medicine, Model Animal Research Center, Medical School of Nanjing University, Nanjing, Jiangsu, China; ${ }^{12}$ Mechanobiology Institute, National University of Singapore, Singapore, Singapore and ${ }^{13}$ Institute of Bioengineering and Nanotechnology, A*STAR, Singapore, Singapore

Correspondence: Han-Ming Shen (phsshm@nus.edu.sg)
}

Published online: 23 July 2018 
we apologize for not detecting them before publication and any inconvenience caused.

Figure S1 PTEN-L resides at the outer mitochondrial membrane. f Co-localization of Flag-PTEN-L with Tom20 without or with CCCP $(5 \mu \mathrm{M}, 4 \mathrm{~h}$ treatment) was examined by confocal fluorescence microscopy. Flag-PTEN-L (Green); Tom20 (Red); co-localization channel (White). Scale bars, 5 and $2 \mu \mathrm{m}$ (ZOOM). i Co-localization of Flag-PTEN-L with Calreticulin. FlagPTEN-L (Green); Calreticulin (Red); co-localization channel (White). Scale bars, 5 and $2 \mu \mathrm{m}$ (ZOOM). 